\title{
Two-Stage Approach for Detection and Reduction of Motion Artifacts in Photoplethysmographic Data
}

\author{
Rajet Krishnan*, Student Member, IEEE, Balasubramaniam (Bala) Natarajan, Senior Member, IEEE, \\ and Steve Warren, Member, IEEE
}

\begin{abstract}
Corruption of photopleythysmograms (PPGs) by motion artifacts has been a serious obstacle to the reliable use of pulse oximeters for real-time, continuous state-of-health monitoring. In this paper, we propose an automated, two-stage PPG data processing method to minimize the effects of motion artifacts. The technique is based on our prior work related to motion artifact detection (stage 1) [R. Krishnan, B. Natarajan, and S. Warren, "Analysis and detection of motion artifacts in photoplethysmographic data using higher order statistics," in Proc. IEEE Int. Conf. Acoust., Speech, Signal Process. (ICASSP 2008), Las Vegas, Nevada, Apr. 2008, pp. 613-616] and motion artifact reduction (stage 2) [R. Krishnan, B. Natarajan, and S. Warren, "Motion artifact reduction in photoplethysmography using magnitude-based frequency domain independent component analysis," in Proc. 17th Int. Conf. Comput. Commun. Network, St. Thomas, Virgin Islands, Aug. 2008, pp. 1-5]. Regarding stage 1, we present novel and consistent techniques to detect the presence of motion artifact in PPGs given higher order statistical information present in the data. We analyze these data in the time and frequency domains (FDs) and identify metrics to distinguish between clean and motion-corrupted data. A Neyman-Pearson detection rule is formulated for each of the metrics. Furthermore, by treating each of the metrics as observations from independent sensors, we employ hard fusion and soft fusion techniques presented in [Z. Chair and P. Varshney, "Optimal data fusion in multiple sensor detection systems," IEEE Trans. Aerosp. Electron. Syst., AES, vol. 1, no. 1, pp. 98-101, Jan. 1986] and [C. C. Lee and J. J. Chao, "Optimum local decision space partitioning for distributed detection,” IEEE Trans. Aerosp. Electron. Syst., AES, vol. 25, no. 7, pp. 536-544, Jul. 1989], respectively, in order to fuse individual decisions into a global system decision. For stage two, we propose a motion artifact reduction method that is effective even in the presence of severe subject movement. The approach involves an enhanced preprocessing unit consisting of a motion detection unit (MDU, developed in this paper), period estimation unit, and Fourier series reconstruction unit. The MDU identifies clean data frames versus those corrupted with motion artifacts. The period estimation unit determines the fundamental frequency of a corrupt frame. The Fourier series reconstruction unit reconstructs the final preprocessed signal by utilizing the spectrum variability of the pulse waveform. Preprocessed data are then fed to a magnitudebased FD independent component analysis unit. This helps reduce motion artifacts present at the frequencies of the reconstruction components. Experimental results are presented to demonstrate the efficacy of the overall motion artifact reduction method.
\end{abstract}

Manuscript received May 6, 2009; revised September 17, 2009 and December 1, 2009; accepted December 1, 2009. Date of publication February 17, 2010; date of current version July 14, 2010. Asterisk indicates corresponding author.

*R. Krishnan was with the Department of Electrical and Computer Engineering, Kansas State University, Manhattan, KS 66506 USA. He is now with the Center of Excellence in Wireless Technology, Indian Institute of Technology Madras, Chennai 600036, India (e-mail: rajetk@ksu.edu).

B. Natarajan and S. Warren are with the Department of Electrical and Computer Engineering, Kansas State University, Manhattan, KS 66506 USA (e-mail: bala@ksu.edu; swarren@ksu.edu).

Digital Object Identifier 10.1109/TBME.2009.2039568
Index Terms-Higher order statistics (HOS), independent component analysis (ICA), magnitude-based frequency-domain ICA (FD-ICA), motion artifact, motion detection unit (MDU), photoplethysmogram (PPG).

\section{INTRODUCTION}

$\mathbf{P}$ HOTOPLETHYSMOGRAPHY is a noninvasive, optical means to obtain relative blood volume in tissue as a function of time. Since hemoglobin is an optical absorber, light passing through tissue is modulated by each cardiac cycle of the subject and also by other processes like respiration and subject motion. The resulting photoplethysmograms (PPGs) can be acquired with reflectance- or transmittance-mode sensors, and multiple excitation wavelengths allow waveform features from time-domain PPGs to be converted into values of blood oxygen saturation. Corruption of PPGs by motion artifacts has been a significant obstruction to efficient and reliable use of pulse oximeters for continuous real-time health monitoring, especially in ambulatory settings [13]. If PPG data are to be reliably obtained from wearable sensors used for real-time, continuous state-of-health monitoring, then effective algorithms for motion artifact reduction must be employed.

Over the years, most of the PPG enhancement research has focused on motion artifact removal techniques. Various signal processing techniques have been investigated to address the problem of recovering quasi-periodic PPGs from data corrupted with motion artifacts. These include wavelet analysis and decomposition techniques [5] and adaptive filters [6]. The study in [7] indicates that both wavelet-transform and adaptive filter techniques introduce phase distortions in PPG data. Generally, PPG distortions due to nonlinear-phase filters do not dramatically affect heart rate (HR) or oxygen saturation ( $\mathrm{SpO} 2)$ calculations. However, problems with nonlinear-phase filters arise when PPG is used for purposes like biometric indicators for patient authentication, where the true waveform shape (without filtering) is important. PPGs are also of interest for comparison with hemodynamic models (e.g., blood pressure/flow models), as they offer a cuffless, noninvasive means to gather these data. In such contexts as well, motion-reduction technique that does not, by nature, distort the target PPG is ideal. Work involving analog filters and moving average techniques is presented in [8]. The artifact extraction problem has also been viewed as a blind source separation problem in [9], [13], and [10]. In [9], an enhanced preprocessing unit preceded the independent component analysis (ICA) block. The preprocessing unit consisted of signal period detection using an autocorrelation method followed by a block-interleaving operation. However, this technique relies 


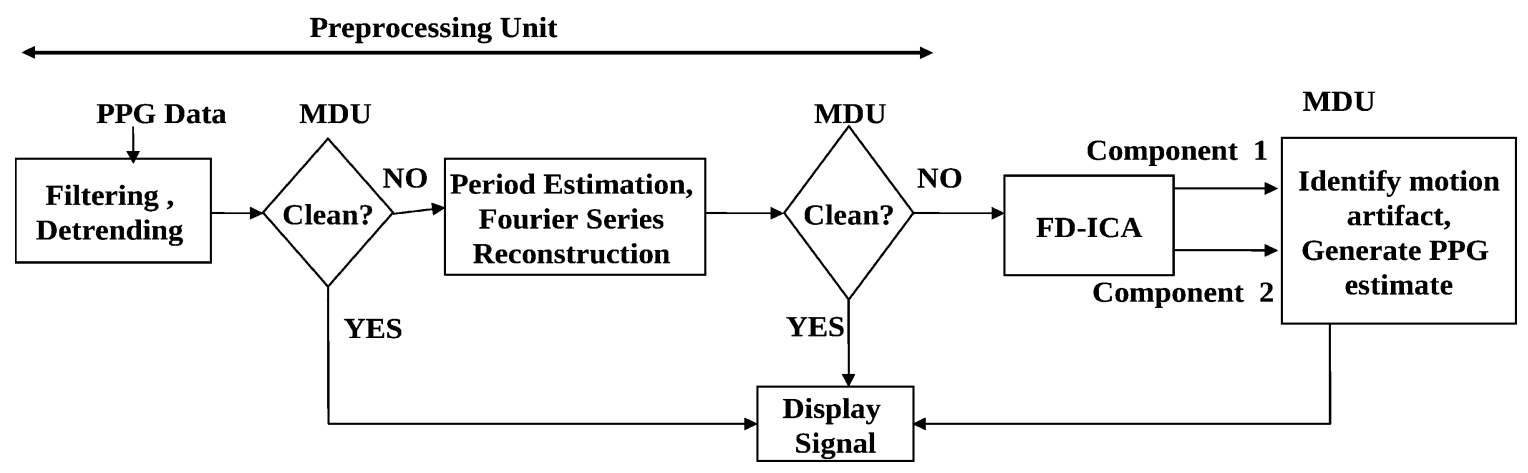

Fig. 1. PPG data processing system model—preprocessing unit (bandpass filter, detrender, MDU, and Fourier series reconstructor), and magnitude-based FD-ICA.

on the ability of the autocorrelation technique to correctly detect the waveform period and hence provides erroneous results in the presence of extreme motion artifacts. In [10], an improved preprocessing technique is described that employs extrapolation/truncation of each cardiac cycle to the mean of the measured cardiac cycle followed by the ICA. This method is highly prone to errors and inconsistencies, since accurate cardiac cycle measurements become difficult in the presence of extreme motion. In [11], a technique based on the use of correlation canceler is described for correlating and canceling motion artifact signals (secondary signal) and obtaining clean PPG signals (primary signal). The primary signal is then used for measuring blood saturation levels and heart-beat rate. However, the accuracy of the technique relies heavily on the ability to accurately determine the proportionality constant and the secondary reference signal.

While removing motion artifact from PPGs is critical, detecting its presence is a key task that needs to be addressed first. A reliable motion artifact detection technique lays the foundation for a completely automated PPG data processing system that can identify PPG data frames contaminated with artifacts and further process them for motion artifact removal. Some work has addressed the detection issue by correlating a PPG data frame with a clean reference signal to detect motion artifact [10]. However, such techniques are unsuitable for robust continuous monitoring where clean PPG signals and motion-corrupted signals are to be identified automatically in real-time implementation.

In this paper, we propose a complete PPG data processing system that combines motion artifact detection and reduction techniques introduced in [1] and [2], respectively. ${ }^{1}$ These two steps are fundamental to the realization of a completely automated PPG processing system that would enable reliable continuous state-of-health monitoring of subjects.

The paper first addresses the issue of detecting the presence of motion artifact based on the inherent time- and frequencydomain (FD) characteristics of PPG data acquired from different subjects. Specifically, the higher order statistics (HOS) properties of clean and motion-corrupted PPG data are distinguishing features that aid detection. In the time domain, skew and kurto-

\footnotetext{
${ }^{1}$ The material in this paper was presented in part at the IEEE Conference on Acoustics, Speech and Signal Processing (ICASSP 2008), Las Vegas, NV, and at the International Conference on Computer Communications and Networks (ICCCN 2008), St. Thomas, Virgin Islands.
}

sis measures associated with the data are analyzed. In the FD, the presence of random components due to motion artifact is ascertained using a FD kurtosis measure as in [21]. Furthermore, bispectral analyses of PPG data indicate the presence of strong quadratic phase coupling (QPC) and more specifically self-coupling in the case of clean PPG data. In motion-artifactcorrupted data, QPC between random frequency components is observed, but the self-coupling feature is absent. NeymanPearson (NP) tests are formulated based on these time-domain and FD metrics. Using practical test data, we characterize the performance (probability of false alarm: $P_{F}$, probability of detection: $P_{D}$, and probability of error: $P_{\text {error }}$ ) of the artifact detection tests. The performance results illustrate the potency of the proposed method for consistent and robust detection of PPG motion artifact. Note that HOS properties of EEG data have been investigated in [12] and [23], and fourth-order cumulants were used in [15] to dynamically determine rhythmic oscillations in PPG data. However, we believe that the paper presented here is the first effort to comprehensively investigate PPG motion artifact detection based on HOS techniques.

As a follow-on to motion artifact detection, we present a new motion artifact reduction method that combines an enhanced signal preprocessing unit and a FD ICA unit. The preprocessing unit incorporates a Fourier series reconstruction of the PPG data that utilizes the spectrum variability and quasi-periodicity of the pulse waveform. Following this is a novel FD-ICA routine that considers only magnitude information. This technique assumes instantaneous mixing of statistically independent sources in the time domain and a constant mixing matrix for the time frame considered. The routine is different from the complex FD-ICA approach described in [24]. A comparison of the technique used in this paper with the time-domain ICA and complex FD-ICA techniques in the literature implies that the new magnitudebased FD ICA approach more effectively reduces motion artifact.

\section{SYSTEM MODEL}

The model for the PPG data processing system is illustrated in Fig. 1 and essentially consists of the preprocessing unit (that consists of the bandpass filter, detrender, MDU, and Fourier series reconstructor) and the FD-ICA unit. PPG data obtained from the pulse oximeter are first filtered and detrended as described 
in Section IV. The motion artifact detection unit (MDU-see Section V), determines whether the data, obtained from filtering and detrending, are corrupt with motion artifacts. If the data are found to be clean, no further cleansing operations are imposed. If the data are corrupt, they are fed into the period estimation and Fourier Series reconstruction unit that yields a PPG signal composed of primarily its fundamental frequency component and harmonics as will be discussed in Section VI. We then use the MDU to determine whether the reconstructed PPG signal is stained with motion artifacts. If the data are not free of motion artifacts, the FD-ICA routine is applied to estimate the blood volume pulsation and motion artifact components (represented by Component 1 and Component 2 in Fig. 1).

\section{THEORY}

The following sections review the HOS measures applied to these PPG data [16]-[20]:

1) Skew and Kurtosis: Skew is a measure of the symmetry (or the lack of it) of a probability distribution, while the kurtosis measure indicates a heavy tail and peakedness $O R$ a light tail and flatness of a distribution relative to the normal distribution. This measure captures the random variations of data from the mean. The skew and kurtosis of a random variable $x$ are given by

$$
\begin{aligned}
C_{3 x}(0,0) & =\frac{\mu_{3}}{\sigma^{3 / 2}} \quad(\text { skew }) \\
C_{4 x}(0,0,0) & =\frac{\mu_{4}}{\sigma^{4}}-3 \quad \text { (kurtosis) }
\end{aligned}
$$

where $\sigma$ is the standard deviation, and $\mu_{3}$ and $\mu_{4}$ are the third- and fourth-central moments, respectively.

2) Bispectrum: The third-order polyspectrum of a random variable $x$ is defined as the Fourier transform of its third cumulant sequence

$$
\begin{aligned}
& S_{3 x}\left(f_{1}, f_{2}\right) \\
& =\sum_{k=-\infty}^{\infty} \sum_{l=-\infty}^{\infty}\left[C_{3 x}(k, l) \exp \left(-j 2 \pi\left(f_{1} k+f_{2} l\right)\right)\right]
\end{aligned}
$$

where $C_{3 x}(k, l)$ is the third cumulant sequence of $x$. The third-order polyspectrum, or the power spectrum, suppresses all phase information in a random process, while the bispectrum does not. When the harmonic components of a process interact, definitive phase relations also exist, in addition to the contribution of power at their sum and difference frequencies; this is called QPC. For example, consider the following process:

$$
\begin{aligned}
X_{1}(k)= & \cos \left(\lambda_{1} k+\phi_{1}\right)+\cos \left(\lambda_{2} k+\phi_{2}\right) \\
& +\cos \left(\lambda_{3} k+\phi_{3}\right)
\end{aligned}
$$

where $\lambda_{3}=\lambda_{1}+\lambda_{2}$, indicating that $\lambda_{1}, \lambda_{2}$, and $\lambda_{3}$ are harmonically related. If $\phi_{1}, \phi_{2}$, and $\phi_{3}$ in (3) are independent random variables uniformly distributed in the range $[0,2 \pi]$, then $\left(\lambda_{3}, \phi_{3}\right)$ is an independent harmonic component. However, if $\phi_{3}=\phi_{1}+\phi_{2}$ in (3), then $\left(\lambda_{3}, \phi_{3}\right)$ is the result of quadratic coupling between $\left(\lambda_{1}, \phi_{1}\right)$ and $\left(\lambda_{2}, \phi_{2}\right)$.

\section{PPG DATA ANALYSIS}

The PPG data analysis is performed to understand and extract features that can be used as distinguishing metrics between clean and motion-corrupted data. Initially, frames of data are collected from healthy subjects in the age group of 22-24 years, using a reflectance pulse oximeter [13], [14]. The subjects follow the same motion patterns as in [13].

1) Stationary position: The subjects remain still with no wrist, finger, or elbow movement.

2) Finger movements (three cases): left-right (swinging), upand down (bending), and arbitrary finger movements while keeping the wrist and elbow stationary.

3) Wrist movements: The wrist is rotated and arbitrarily moved, keeping the elbow and fingers stationary.

4) Elbow movements: The elbow is bent and extended, keeping the wrist and fingers stationary.

Data are fed into a MATLAB script that partitions the entire data segment into short frames of equal length. First, each frame is passed through a bandpass filter $(0.3-12 \mathrm{~Hz})$. Here, the design of the filter is critical, as the phase information in the data needs to be preserved to retain the shape of the PPG waveform. For this purpose, a zero-phase forward-reverse filter of order four in both directions is chosen. After filtering, the baseline trend associated with each data frame is removed by extracting an appropriately fitted polynomial curve. Each data frame is then inspected in the time domain and FD, and the HOS properties are characterized.

\section{A. Time-Domain Analysis}

In the time domain, we analyze the skew and kurtosis measure of the time variation of the amplitude of the PPG signal in each frame considered. This is done by evaluating (1) for each data frame. It is important to note that these measures will vary with age and health condition. It is observed that the skew and kurtosis measured for the case of motion-corrupted data are much higher in magnitude when compared to the skew and kurtosis for clean data. Therefore, these measures could serve as candidate features for motion detection.

\section{B. FD Analysis}

In the FD, the kurtosis measure is computed for the magnitude of the Fourier spectrum for each data frame. This measure considers the magnitude of the components present at each frequency sampled by the discrete Fourier transform operation. It is seen that kurtosis is lesser in magnitude for frames corrupted with motion artifact versus frames with clean data. This means that a Fourier spectrum of clean data has a lower number of significant frequency components (since only the harmonic components are prominent) compared to a spectrum of motion corrupted data (that consists of harmonic and random spectral components).

\section{Bispectral Analysis and QPC}

The bispectrum and the bicoherence of each data frame are analyzed using the MATLAB Higher-Order Spectral Analysis 
TABLE I

BisPeCTRUM PLOT RESUlTS: CLEAN DATA

\begin{tabular}{c|c}
\hline Coupling Frequency $(f) \mathrm{Hz}$ & Coupling Magnitude \\
\hline \hline 1.54 & 0.1565 \\
\hline 3.08 & 0.0211 \\
\hline 4.62 & 0.0037 \\
\hline
\end{tabular}

TABLE II

BisPECTRUM PLOT RESUlTS: CORRUPT DATA

\begin{tabular}{c|c}
\hline Coupling Frequency $(f) \mathrm{Hz}$ & Coupling Magnitude \\
\hline \hline 0.74 & 0.2839 \\
\hline 2.74 & 0.0081 \\
\hline 4.41 & 0.0009 \\
\hline
\end{tabular}

Toolbox [18]. Significant peaks at nonzero frequencies are observed in the bispectrum diagonal slice plots for clean PPG data, thereby confirming the presence of strong QPC. In the case of clean PPG data from the initial subjects, Table I indicates peaks at $t f_{0} \mathrm{~Hz}, t=1,2,3$, where $f_{0}=1.54 \mathrm{~Hz}$ is the most dominant frequency being coupled, indicating the presence of self-coupling between frequencies (we have $f_{0}+f_{0}=2 f_{0}$ and $f_{0}+2 f_{0}=3 f_{0}$ and so on, indicating equally spaced peaks in the diagonal slice plot). However, in the case of corrupt PPG data, QPC is observed to occur between random frequency components, and the phenomenon of self-coupling is absent, as illustrated in Table II. The features used for motion detection are summarized shortly

1) Time-domain features: Skew and kurtosis measures that provide information on the distribution of data. They contain information regarding amplitude variation of the PPG waveform.

2) FD feature: FD kurtosis measure that indicates the presence of random components in the Fourier spectrum, which are not present in the spectrum of a clean signal that contains only the main harmonics.

3) Bispectral feature and QPC: Clean PPG data are characterized by the presence of strong self-coupling between the fundamental components of the frequency spectrum. This is absent in artifact-corrupted measurements, where QPC between random frequency components is observed.

\section{Motion Detection Unit}

\section{A. Methods for Motion Artifact Detection}

1) Neyman-Pearson Detection Rule Formulation: We adopt NP Detection method [22] for detecting motion artifacts in PPG measurements. PPG data were collected from ten healthy male and female subjects, in the age group of 22-30 years (different subjects from those considered for preliminary analyses and feature extractions in Section IV), in order to formulate the hypotheses for the NP detection rule. The subjects followed the same motion routines as detailed in Section IV. Based on the resulting data, distinguishing measures were computed for each data frame as described in Section IV. For each of the measures, let $H_{0}$ denotes the null hypothesis corresponding to the region for clean data and $H_{1}$ denote the alternative hypothesis corresponding to the region for corrupt data. Under the hypotheses
$H_{0}$ and $H_{1}$ the time-domain kurtosis, skew, and FD kurtosis measures are distributed as

$$
\begin{array}{ll}
H_{0}: \quad y_{i} \sim \mathcal{N}\left(\mu_{0 i}, \sigma_{0 i}^{2}\right) \\
H_{1}: \quad y_{i} \sim \mathcal{N}\left(\mu_{1 i}, \sigma_{1 i}^{2}\right) \quad \forall i \in\{1,2,3\}
\end{array}
$$

where $\mathcal{N}\left(\mu, \sigma^{2}\right)$ is a Gaussian distribution with mean $\mu$ and variance $\sigma^{2}$, and $i$ corresponds to each of the distinguishing metrics.

$y_{i}$ is the observation corresponding to the time-domain kurtosis $(i=1)$, skew $(i=2)$, and FD kurtosis $(i=3)$ measures. The sample histogram plots of clean (hypothesis $H_{0}$ ) and noisy (hypothesis $H_{1}$ ) time-domain kurtosis measure data are provided in Fig. 2. Based on values of the time-domain kurtosis and skew for each frame, local decisions $\delta_{i} \in\{-1,1\}$ are made according to

$$
\delta_{i}=\left\{\begin{array}{ll}
1 & \text { if } y_{i} \geq \eta_{i} \\
-1 & \text { if } y_{i}<\eta_{i}
\end{array} \text { for } i \in\{1,2\}\right.
$$

where $\eta_{i}=\sigma_{0 i} \mathcal{Q}^{-1}\left(1-P_{F_{i}}\right)+\mu_{0 i}, \delta_{i}=-1$ corresponds to the null hypothesis, and $\delta_{i}=1$ corresponds to the alternative hypothesis, $\forall$ is "for all," $\in$ is of set."Here, $P_{F_{i}}$ is the false-alarm probability. For FD kurtosis, a decision is made according to

$$
\delta_{i}=\left\{\begin{array}{ll}
1 & \text { if } y_{i} \leq \eta_{i} \\
-1 & \text { if } y_{i}>\eta_{i}
\end{array} \quad \text { for } i=3\right.
$$

where $\eta_{i}=\sigma_{0 i} \mathcal{Q}^{-1}\left(P_{F_{i}}\right)+\mu_{0 i}$. It can be easily shown for the time-domain kurtosis and skew measures that

$$
\begin{aligned}
& P_{F_{i}}=1-\mathcal{Q}\left(\frac{\eta_{i}-\mu_{0 i}}{\sigma_{0 i}}\right) \text { and } \\
& P_{D_{i}}=1-\mathcal{Q}\left(\frac{\eta_{i}-\mu_{1 i}}{\sigma_{1 i}}\right)
\end{aligned}
$$

where $P_{D_{i}}$ is the corresponding probability of detection for each measure, and $\mathcal{Q}^{-1}$ is the inverse $\mathcal{Q}$-function where, $\mathcal{Q}$-function is the tail probability of the standard normal distribution. For the FD kurtosis measure, the corresponding $P_{F_{i}}$ and $P_{D_{i}}$ are given by

$$
\begin{aligned}
P_{F_{i}} & =\mathcal{Q}\left(\frac{\eta_{i}-\mu_{0 i}}{\sigma_{0 i}}\right) \text { and } \\
P_{D_{i}} & =\mathcal{Q}\left(\frac{\eta_{i}-\mu_{1 i}}{\sigma_{1 i}}\right) .
\end{aligned}
$$

The tests in (5) and (6) are applied to data obtained from three new healthy test subjects of 22-30 years of age [different subjects from those considered for formulating the hypotheses in (4)], in addition to the data from ten subjects considered earlier. The performance of all motion detectors using test data from the three new subjects (for brevity of space) is demonstrated against its theoretical performance, using receiver operating characteristic (ROC) curves [22] in Fig. 3. It is important to note that the performance on test data conforms to that expected in theory, assuming a Gaussian distribution for $y_{i}$. The kurtosis measures in the time domain and FD perform better than the skew measure in the time domain. This is because the skew measure indicates the symmetry (or the lack of it) of the distribution of the data about the mean and is thus more characteristic of the PPG waveform 


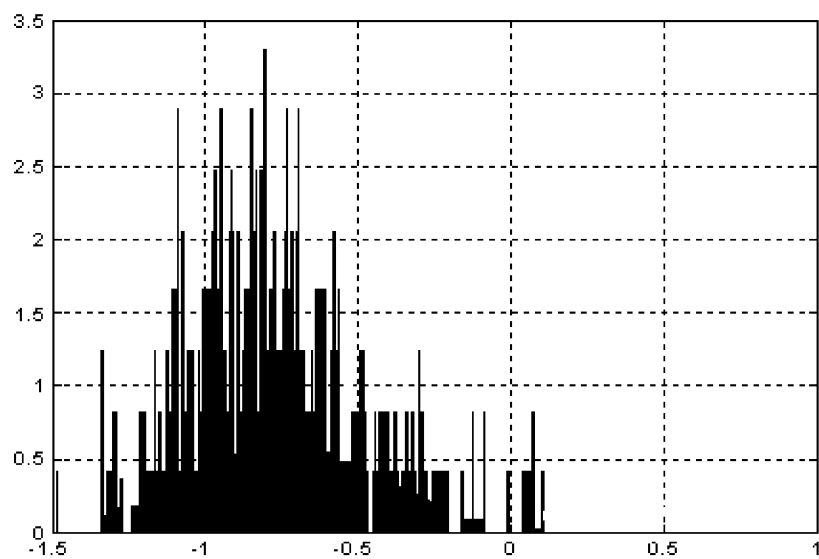

(a)

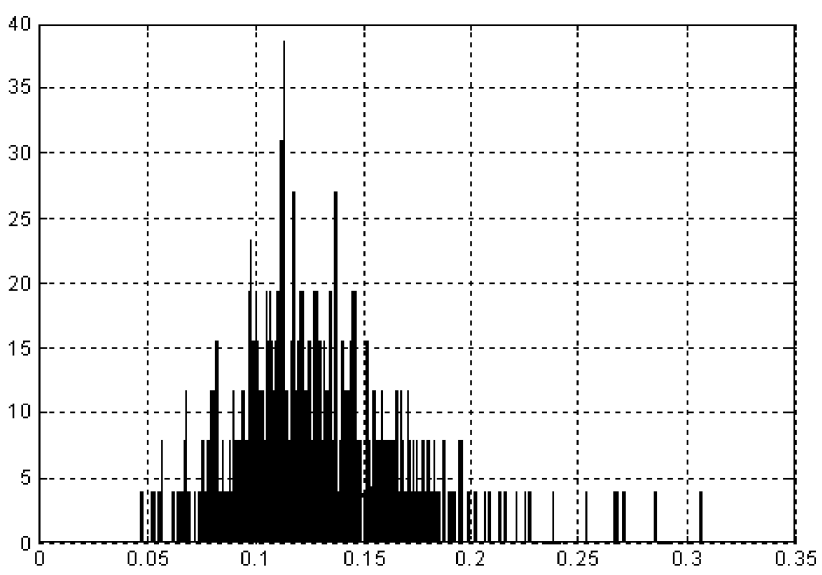

(b)

Fig. 2. Sample histogram plots of (a) clean (hypothesis $H_{0}$ ) and (b) noisy (hypothesis $H_{1}$ ) time-domain kurtosis measure data.

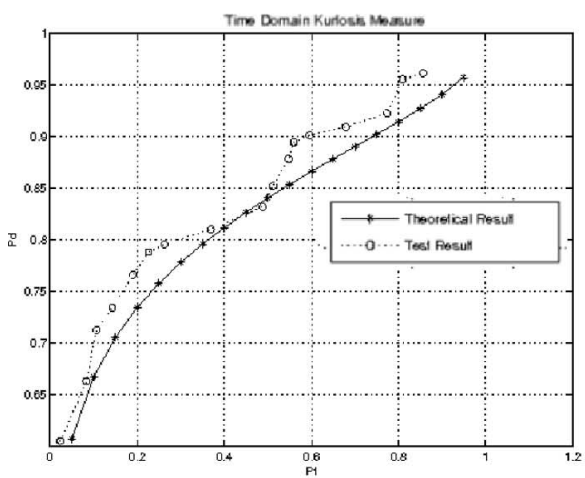

(a)

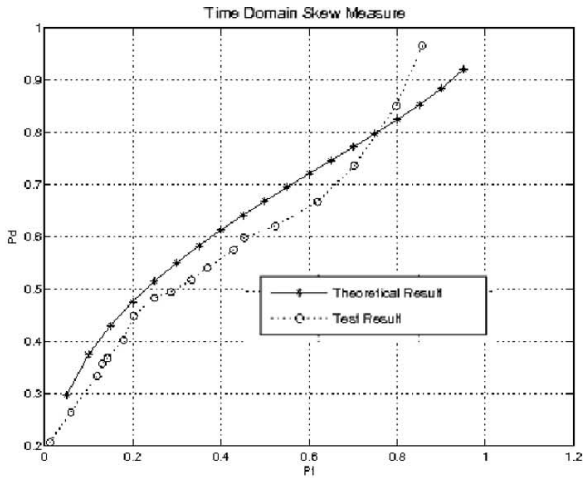

(b)

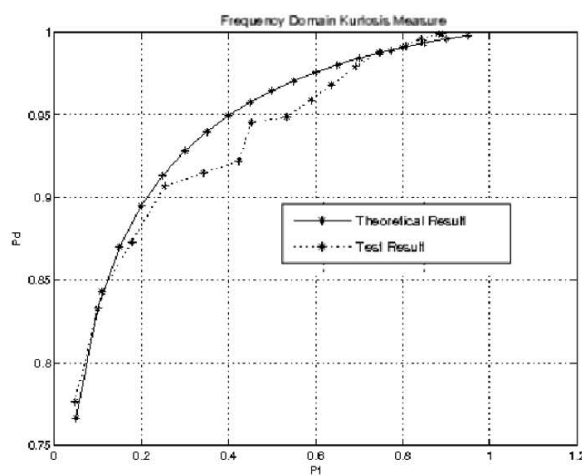

(c)

Fig. 3. ROC curves (theoretical plot versus test data plot) for the (a) time-domain kurtosis measure, (b) time-domain skew measure, and (c) FD kurtosis measure.

(or the subject), while the kurtosis measure captures random variations from the mean.

2) Self-Coupling Detection Rule: As concluded earlier, clean PPG data are characterized by self-coupling, which is absent from data containing motion artifact (though QPC between random frequency components is present). That is, self-coupling implies that the data are clean or contain insignificant amount of motion artifacts. Hence, to determine the presence/absence of self-coupling, the frequencies being coupled are noted for each data frame and a decision is made as follows:

$$
\delta_{i}= \begin{cases}1 & \text { Self-coupling } \Rightarrow \text { clean data } \\ -1 & \text { No self-coupling } \Rightarrow \text { corrupt data. }\end{cases}
$$

The $P_{D}$ and $P_{F}$ measures related to the self-coupling measure are directly computed from the initial training set. The $P_{F}$ value is found to be 0.0420 , while the $P_{D}$ value is found to be 0.8932 for this training set.

\section{B. Decision Fusion}

The time-domain measures (kurtosis, skew) and the FD measures (QPC, kurtosis) are modeled as four individual sensors, whose independent decisions can be fused to detect the presence of motion artifact in a given data frame. To implement this sensor decision fusion, we employ two methods: hard-decision fusion presented in [3] and soft-decision fusion developed in [4].

The hard-decision fusion technique fuses individual sensor decisions while minimizing the probability of error for the overall detection system. Weights or reliability measures that are a function of individual $P_{F_{i}}$ and $P_{D_{i}}$ values are associated with the decisions made by the individual sensors, and the fused global decision is given as follows:

$$
f\left(\delta_{1}, \ldots, \delta_{n}\right)= \begin{cases}+1 & \text { if } a_{0}+\sum_{i=1}^{4} a_{i} \delta_{i}>0 \\ -1 & \text { otherwise }\end{cases}
$$

where $\delta_{i}=+1$ and $\delta_{i}=-1 \forall i \in\{1,2,3,4\}$ are the decisions made by the individual sensors corresponding to the presence/absence respective of motion artifact based on the detection 
TABLE III

SENSOR-DECISION-FUSION RESULTS

\begin{tabular}{c|c|c|c|c|c|c}
\hline Sensor & $P_{F}$ & $P_{D}$ & $P_{\text {error }}$ & $P_{F}$ & $P_{D}$ & $P_{\text {error }}$ \\
\hline \hline Kurtosis & 0.2 & 0.78 & 0.16 & 0.4 & 0.85 & 0.19 \\
\hline Skew & 0.2 & 0.42 & 0.40 & 0.4 & 0.58 & 0.47 \\
\hline QPC & 0.04 & 0.89 & 0.08 & 0.04 & 0.89 & 0.08 \\
\hline FDK & 0.2 & 0.9 & 0.2 & 0.4 & 0.92 & 0.25 \\
\hline Fused Decision (hard) & 0.07 & 0.91 & 0.07 & 0.2 & 0.94 & 0.11 \\
\hline Fused Decision (soft) & 0.06 & 0.92 & 0.06 & 0.22 & 0.96 & 0.14 \\
\hline
\end{tabular}

rules developed in Section V-A. The weights $a_{i}$ are defined as

$$
\begin{aligned}
& a_{0}=0 \\
& a_{i}=\log \left(\frac{P_{D_{i}}}{P_{F_{i}}}\right) \quad \text { if } \delta_{i}=+1 \\
& a_{i}=\log \left(\frac{1-P_{F_{i}}}{1-P_{D_{i}}}\right) \quad \text { if } \delta_{i}=-1
\end{aligned}
$$

assuming uniform cost assignment and equal prior probabilities for both hypotheses in (4).

The tests in (5), (6), and (9) are applied to data obtained from three test subjects as described in the previous section to obtain $\delta_{i} \forall i \in\{1,2,3,4\}$. We select thresholds in (5) and (6) to yield $P_{F_{i}}=0.2$. We then evaluate the individual $P_{D_{i}}$ and their respective probability of error $P_{\text {error }_{i}}$. The weights are computed as in (11), and the fused decision is formed using (10). This is repeated for $P_{F_{i}}=0.4$.

Under the same assumption of uniform costs and equal prior probabilities, we employ the soft fusion technique as in [4]. In this technique, we partition each of the hypothesis regions $H_{0}$ and $H_{1}$ into mutually exclusive subregions and associate a level of confidence with each of them. The level of confidence depends on the distance of the local decision statistic from the decision threshold and hence is also a function of the probability of false alarm and the probability of detection associated with the subregion. Optimal partitioning of the local decision space is achieved by partitioning the probability of false alarm and the probability of detection based on a $J$-divergence maximization criterion. For the time-domain kurtosis and skew measures, $P_{F_{i}}$ and $P_{D_{i}}$ have a functional relationship from (7) of

$$
P_{D_{i}}=1-\mathcal{Q}\left(\frac{\sigma_{0 i} \mathcal{Q}^{-1}\left(1-P_{F_{i}}\right)+\mu_{0 i}-\mu_{1 i}}{\sigma_{1 i}}\right), \quad i \in\{1,2\}
$$

For the FD kurtosis measure, the relationship between $P_{F_{i}}$ and $P_{D_{i}}$ from (8) is as follows:

$$
P_{D_{i}}=\mathcal{Q}\left(\frac{\sigma_{0 i} \mathcal{Q}^{-1}\left(P_{F_{i}}\right)+\mu_{0 i}-\mu_{1 i}}{\sigma_{1 i}}\right), \quad i=3
$$

The soft-decision fusion technique is performed for $P_{F_{i}}=0.2$ and $P_{F_{i}}=0.4$ for $i \in\{1,2,3\}$. We refer the readers to [4] for a detailed qualitative treatment.

The results are summarized in Table III. It can be easily seen that for both values of $P_{F_{i}}$, the fused decision, and in particular the soft fusion technique, provides a better probability of detection of motion artifact than the individual sensors.

\section{Motion ARtifact Reduction Method}

The proposed motion artifact reduction method consists of a preprocessing unit and an FD-ICA unit. The preprocessing unit employs a bandpass filter, detrender, MDU, and Fourier series reconstruction, and its output is fed into the FD-ICA unit. The FD-ICA unit separates out the motion artifacts present at the frequency components chosen for reconstruction.

\section{A. Preprocessing Unit}

The preprocessing unit consists of the detrender, bandpass filter, MDU, period estimator, and Fourier series reconstructor, as shown in Fig. 1. The detrender, bandpass filter, and MDU are the same as those used in Sections IV and V, respectively.

1) Period Estimation: The period of the PPG signal can be estimated by an autocorrelation operation. However, accurate and consistent prediction of the period is not possible by this method in the presence of extreme motion artifacts, where the PPG data are completely buried in noise. Hence, an indirect method for the computation of the period is considered (using the MDU from Section V). When a frame with motion artifact is identified as corrupt, the most recent frame with clean data is identified. The most significant frequency component from the clean frame is identified from its Fourier spectrum and also used as the fundamental period for the corrupt frame. This is a reasonable assumption, since moment-to-moment changes in HR should be minor.

2) Fourier Series Reconstruction: Upon obtaining the fundamental frequency and its harmonics for a corrupt frame, a Fourier series reconstruction of the signal is performed. In this reconstruction process, frequencies in the neighborhood of the harmonics are also used to account for the spectral variability of the PPG data. This is motivated by the inherent quasi-periodic nature of PPG signals. Additionally, in the presence of motion artifact, we expect a possible doppler spread around the fundamental frequency and its harmonics. Hence, we must account for this frequency-dependent spread of energy. We use roughly 5-to-6 significant frequency components near the fundamental frequency determined for the data frame under consideration. The number of frequency components chosen is a tradeoff decision that would depend on several factors-using greater number of power components from the Fourier spectrum would render the preprocessed signal to be heavily noisy due to increase in contribution from motion artifacts. At the same time, power contribution from a clean PPG may have widened in frequency, depending on the intensity and nature of the movement artifact. Therefore, the neighborhood decision (i.e., the number of frequency components around the fundamental frequency) is a designers choice that must be based on a careful consideration of all factors. The technique is illustrated in Fig. 4.

\section{B. FD-ICA Unit}

In this paper, we assume that motion artifacts and PPG signal sources mix linearly with a mixing matrix in the time domain to form the observables (measurements). The observables, denoted by $x_{1}(t)$ and $x_{2}(t)$, are the preprocessed measurements due 


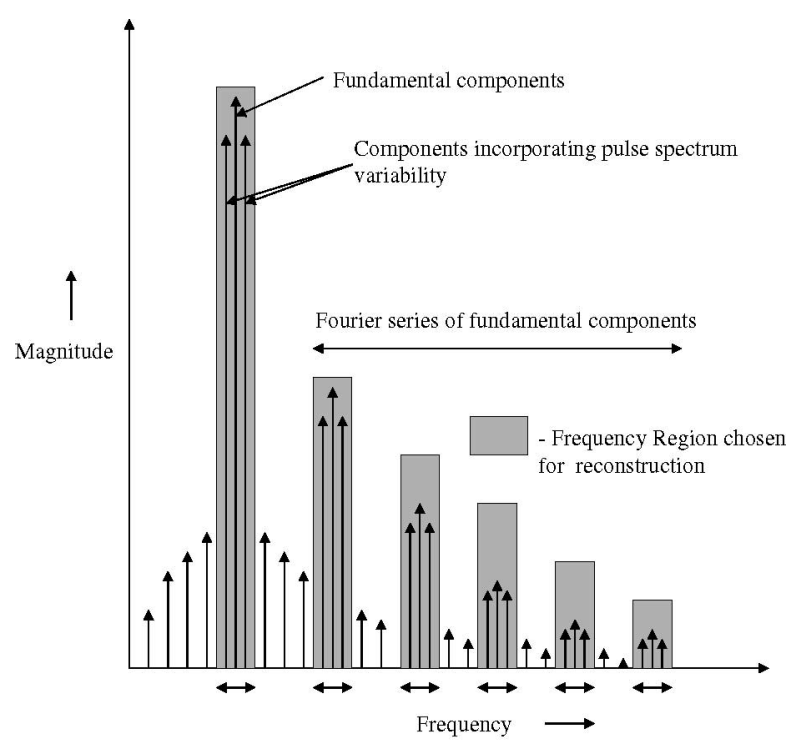

Fig. 4. Fourier series reconstruction of a motion-corrupted frame.

to the near-IR and red excitation sources, respectively. For a measurement time frame $\tau$, this instantaneous mixing is given by

$$
\begin{aligned}
& x_{1}(t)=a_{11} s_{1}(t)+a_{12} s_{2}(t) \\
& x_{2}(t)=a_{21} s_{1}(t)+a_{22} s_{2}(t)
\end{aligned}
$$

where sources $s_{1}(t)$ and $s_{2}(t)$ denote the time-domain PPGs and motion artifacts, respectively. The mixing matrix $\mathbf{A}$ is assumed to be constant over the time frame $\tau$. Hence, the FD representation of (12) is

$$
\begin{aligned}
& X_{1}(f)=a_{11} S_{1}(f)+a_{12} S_{2}(f) \\
& X_{2}(f)=a_{21} S_{1}(f)+a_{22} S_{2}(f)
\end{aligned}
$$

where $X_{i}(f)$ and $S_{i}(f)$ are the Fourier transforms of $x_{i}(t)$ and $s_{i}(t)$ for $i=1$ and 2. Now, considering the magnitude of $X_{i}(f)$ and using the triangle inequality, we can write (13) as

$$
\begin{aligned}
& \left|X_{1}(f)\right| \leq a_{11}\left|S_{1}(f)\right|+a_{12}\left|S_{2}(f)\right| \\
& \left|X_{2}(f)\right| \leq a_{21}\left|S_{1}(f)\right|+a_{22}\left|S_{2}(f)\right| .
\end{aligned}
$$

Both heart activity and motion artifacts affect the blood vessel volume at the tip of the finger, the acquisition point for PPG data in this study. Variations in blood volume due to source interference are observed to result in corrupt PPG data. However, interference between the two source signals is maximal when they are aligned in the same direction in the signal space. That is, for the case of maximal interference, $S_{1}(f)$ and $S_{2}(f)$ exhibit linear dependence, thus equality in (14) can be considered. Therefore,

$$
\begin{aligned}
& \left|X_{1}(f)\right| \approx a_{11}\left|S_{1}(f)\right|+a_{12}\left|S_{2}(f)\right| \\
& \left|X_{2}(f)\right| \approx a_{21}\left|S_{1}(f)\right|+a_{22}\left|S_{2}(f)\right| .
\end{aligned}
$$

Hence, the Fourier magnitude spectrum of the corrupt PPG data can be modeled as motion artifacts and pulsatile blood volume components linearly mixing with an unknown mixing matrix.
Since these pulsatile signals and motion artifacts are assumed to be statistically independent, we can employ ICA in either the time domain or FD. From (15), ICA can be performed on the magnitude spectrum of $x_{1}(t)$ and $x_{2}(t)$, using the fastICA MATLAB package based on the fast ICA algorithm [25]. After applying the ICA routine to these magnitude spectra, we obtain an estimate of $\hat{S}_{1}(f)$ and $\hat{S}_{2}(f)$ that represents blood volume pulsation and motion artifact magnitude information, respectively. Utilizing the phase information of the original PPG data, we then reconstruct the clean PPG data and the motion artifacts.

In traditional FD ICA approaches [24], ICA is performed on complex data under the assumption that the mixing matrix is different for each frequency bin. In another FD approach applied to speech recognition [26], the energy of the observables in the FD is considered, but the unmixing matrix varies for different frequency bins. As a result, conventional approaches suffer from permutation problems and gain issues as discussed in [24]. However, these issues do not exist in our approach since all of the frequencies selected by the preprocessing unit are treated as a single bin for which an unmixing matrix is computed, i.e., the mixing/unmixing matrix is treated as frequency nonselective (constant for all frequencies). The gain issue is tackled by normalizing the determinant of the unmixing matrix to unity, as discussed in [24], then the power of the recovered PPG source signal (obtained after the FD-ICA process) is scaled to the original PPG measurement data.

\section{METHODS}

Data were collected from ten healthy subjects in the age group of 22-30 years, who were subjected to the same motion routine as mentioned in Section IV. Data from each subject were fed to a MATLAB script that dissects the entire segment into short equal-length frames. The frame length was picked to accommodate roughly three-to-four heart cycles in each of the frames, implying a time length of about 2-3 s. One factor considered for this purpose was to keep data frames short enough such that the mixing matrix can be assumed to be a constant across the data frame considered (stationarity concern in ICA); yet long enough to derive crucial statistical and spectral information relevant to the frame under consideration. It may be noted that determining the optimal frame length is a design issue in itself that would have to incorporate factors like speed or frequency of motion (that would help determine the accurate time length over which the mixing matrix may be regarded constant) and physiological factors. These frames were fed to the MDU to detect the presence of motion artifact. The frames identified as corrupt were then processed using the technique described in Section VI.

\section{RESULTS AND DISCUSSION}

The results of the separation process for a single subject are presented in Fig. 5. Each recovered signal segment is visually compared (shape and peak-to-peak amplitude) with the most recent clean frame (the Reference). The proposed technique (magnitude-based FD-ICA) is effective even in the presence of significant motion artifacts. 
TABLE IV

CORRELATION COEFFICIENT FOR QUANTITATIVE COMPARISON OF DIFFERENT TECHNIQUES

\begin{tabular}{|c|c|c|c|c|c|}
\hline & Wrist Movement & Finger (left/right) & Finger (up/down) & Elbow Movement & Random Movement \\
\hline Time Domain ICA & 0.69 & 0.67 & 0.63 & 0.65 & 0.66 \\
\hline Magnitude-based FD-ICA & 0.76 & 0.72 & 0.69 & 0.71 & 0.74 \\
\hline Complex ICA & 0.61 & 0.57 & 0.43 & 0.57 & 0.59 \\
\hline Fourier Preprocessing & 0.52 & 0.50 & 0.41 & 0.50 & 0.52 \\
\hline
\end{tabular}
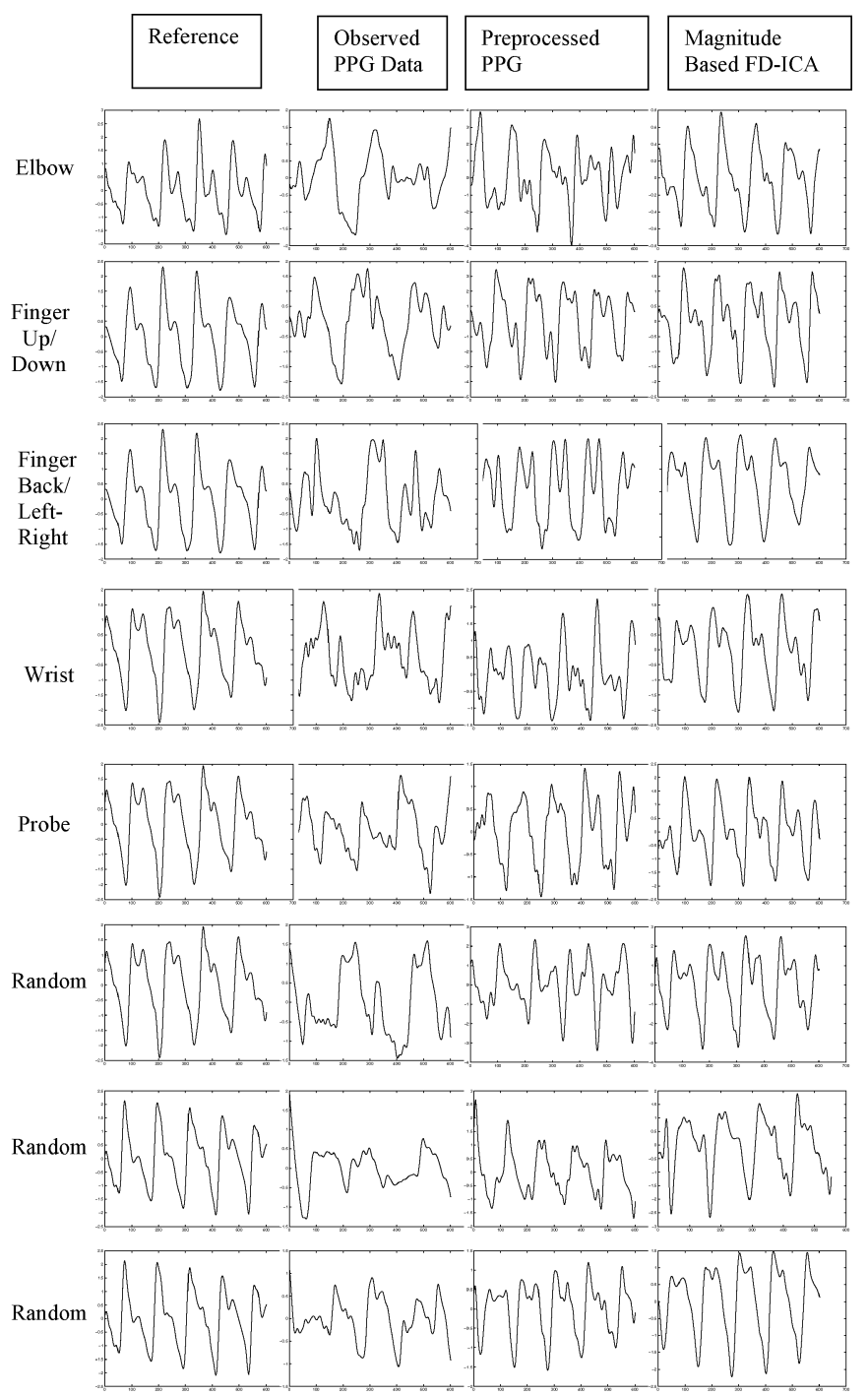

Fig. 5. Separation results using the new technique-sampling rate of pulse oximeter: $200 \mathrm{~Hz}$; frame length: 2-3 s; reference PPG (clean); observed PPG (measured); preprocessed PPG (after detrending, filtering, and Fourier series reconstruction); magnitude-based FD-ICA.

A quantitative comparison between various techniques as applied to different types of motion artifacts is presented in Table IV. A correlation coefficient (CC) (normalized to unity) is obtained by identifying the maximum of all peaks that appear in the normalized cross-correlation plot between the chosen reference signals and the output signals recovered by each of the techniques.

The same separation routine, when applied to data obtained from the rest of the ten subjects, effectively recovers the clean PPG data from the corrupt frames in all cases. However, the efficacy of the whole routine depends primarily on the preprocessing phase, in particular the accuracy in determining the fundamental frequency of the corrupt frame. This can be seen from Table IV, where the CCs between the recovered signals and their corresponding references drop when recovery from the Fourier preprocessing phase is not effective enough. Hence, it can be understood that the clean signal are recoverable if the fundamental frequency, determined from the most recent clean frame (reference), matches the actual fundamental frequency of the corrupt frame.

\section{A. Comparison Between FD-ICA and Time-Domain ICA Methods}

Assuming the independence of source signals and their linear mixing with an unknown mixing matrix in the time domain as in (12), the preprocessed observables were sent to a timedomain ICA routine. The results obtained from the time-domain ICA routine were then visually compared with those from the FD-ICA technique and the reference signal (shape and peakto-valley height), as shown in Fig. 6. The FD-ICA technique outperforms the time-domain ICA process. This is also apparent from Table IV: the CC associated with the FD-ICA routine is much higher than its time domain counterpart for all cases of movement. It may be noted that when performing an ICA based on the assumption of a constant mixing/unmixing matrix, the duration of the data frame should be short enough for the assumption to hold. Using long data frames would imply that the mixing/unmixing matrix is not a constant and hence leads to inaccurate estimation of the sources.

\section{B. Comparison Between FD-ICA and Complex ICA Methods}

In the complex ICA formulation of the problem, it is assumed that the sources mix with the unknown mixing matrix in a convolutive manner in the time domain. This directly translates to the instantaneous mixing of the sources with the mixing matrix in the FD as in [24]. Here, the assumption of independence between the sources in the FD is considered. For fairness in comparison, all frequencies selected by the preprocessing unit are treated as a single frequency bin. The mixing matrix is assumed to be frequency nonselective, and hence only one mixing matrix is computed for all the frequencies selected during preprocessing, unlike the traditional practice adopted in the complex FD ICA approach. The complex ICA routine described in [27] was implemented for the complex data obtained by the Fourier transform of each preprocessed signal. The results obtained from the complex FD ICA routine were visually compared with those from the FD-ICA technique and the reference signal (shape and peak-to-valley height) in Fig. 6. It can be easily seen that the newly proposed FD-ICA routine outperforms the complex ICA 

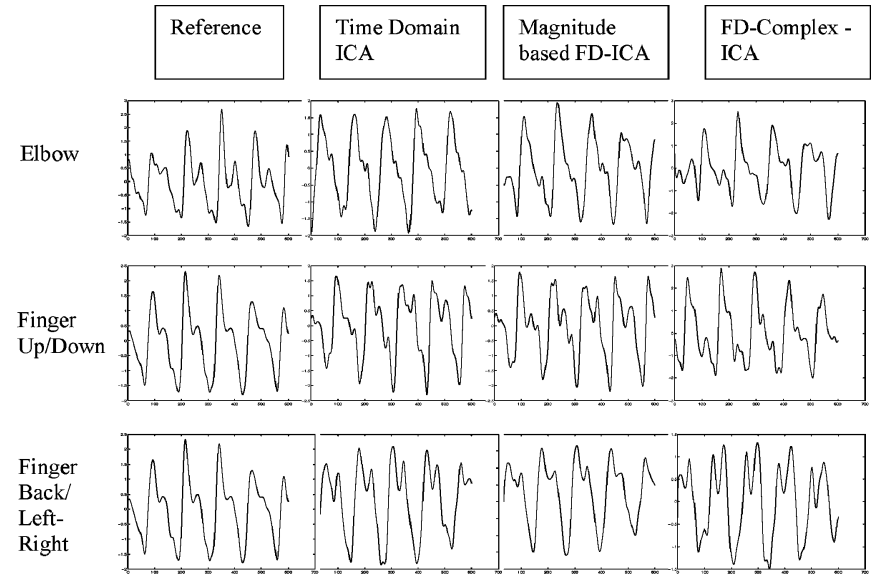

Wrist
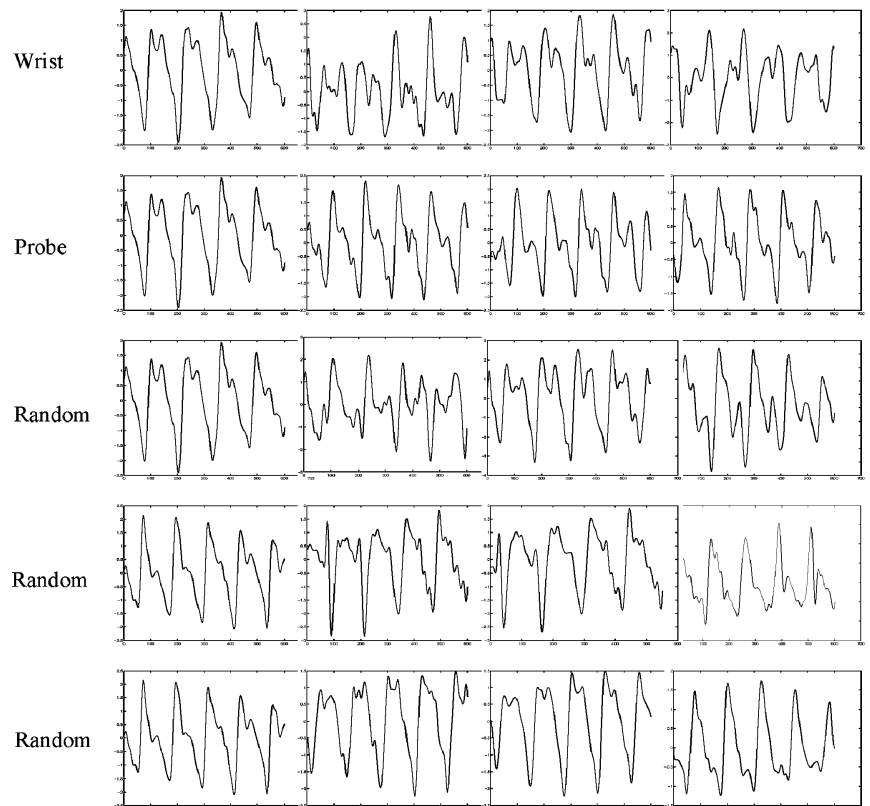

Fig. 6. Comparison of the FD-ICA techniques with the time-domain ICA and complex ICA approaches.

routine. The superiority of the proposed technique is quantified in Table IV: the CC for the FD-ICA method is consistently higher than that of the complex ICA approach for all cases of movement.

\section{CONCLUSION}

In this paper, we formulate a robust method for detection and removal of motion artifact in PPG data, primarily considering HOS in the detection phase. In the time domain, we observe that the skew and kurtosis measures associated with the motioncorrupted PPG data are much higher in magnitude than the same measures for clean PPG data. The FD kurtosis measure is much smaller for the corrupt data frames than for the clean frames. Bispectral analyses of PPG data indicate the presence of strong QPC and, more specifically, self-coupling in the case of clean PPG data. Though QPC is found in data corrupted by motion artifacts, the self-coupling feature for the desired PPG is absent. Based on all of these observations, NP rules are formulated for each of the measures. It is understood that kurtosis-based detec- tion is more reliable than the skew measure. It is seen that softdecision fusion based on individual measures further enhances the overall detection capability. In summary, the HOS-based motion detection algorithm is a consistent and reliable method to identify corrupt data frames that can be further processed for motion artifact removal.

For practical implementation, motion artifact detection maybe be performed with a cognitive sensor that would operate in two phases.

1) Learning phase: In this phase, the sensor unit determines the time domain and FD measures associated with clean and motion-artifact-corrupted PPG signal of the subject. Then the NP rules are formulated based on observations in the learning phase. This process can be perceived as something similar to how a voice recognition software is set up on a cell phone.

2) Operational phase: The sensor determines the frames that are corrupt and clean based on the formulated NP rules. Once a frame is identified to have motion artifacts, artifact reduction/removal algorithms are initiated to clean the signal.

In this implementation model, once the learning phase is complete, the sensor (along with the motion artifact detection and removal algorithms) can operate in real time. We acknowledge that learning may render this technique unattractive for routine clinical use with existing off-the-shelf devices. However, we presume that with advances in sensor processing power and minor updates to device architectures (e.g., secondary processing chips), the computational loads incurred and the processing times required for learning would be significantly reduced.

In addition to the MDU, this paper describes an enhanced PPG preprocessing routine that is employed prior to the magnitudebased FD ICA routine. We readily observe that this processing routine effectively reduces motion artifacts in corrupt data frames even in the event of significant motion. The FD-ICA routine proposed in this paper is compared with time-domain ICA and complex ICA routines and is shown to be more effective in recovering clean PPG data. The efficacy of the method depends heavily on the ability of the MDU to identify corrupt/clean data segments and estimate the period of the waveform. More accuracy in the fundamental period estimation of the corrupt frame helps the FD-ICA routine to more effectively separate motion artifacts from desired data.

\section{REFERENCES}

[1] R. Krishnan, B. Natarajan, and S. Warren, "Analysis and detection of motion artifacts in photoplethysmographic data using higher order statistics,' in Proc. IEEE Int. Conf. Acoust., Speech, Signal Process. (ICASSP 2008), Las Vegas, Nevada, Apr. 2008, pp. 613-616.

[2] R. Krishnan, B. Natarajan, and S. Warren, "Motion artifact reduction in photoplethysmography using magnitude-based frequency domain independent component analysis," in Proc. 17th Int. Conf. Comput. Commun. Network, St. Thomas, Virgin Islands, Aug. 2008, pp. 1-5.

[3] Z. Chair and P. Varshney, "Optimal data fusion in multiple sensor detection systems," IEEE Trans. Aerosp. Electron. Syst., AES, vol. 1, no. 1, pp. 98 101, Jan. 1986

[4] C. C. Lee and J. J. Chao, "Optimum local decision space partitioning for distributed detection," IEEE Trans. Aerosp. Electron. Syst., AES, vol. 25, no. 7, pp. 536-544, Jul. 1989. 
[5] S. Lee, B. L. Ibey, W. Xu, M. A. Wilson, M. N. Ericson, and G. L. Cote, "Processing of pulse oximeter data using discrete wavelet analysis," IEEE Trans. Biomed. Eng., vol. 52, no. 7, pp. 1350-1352, Jul. 2005.

[6] J. M. Graybeal and M. T. Petterson, "Adaptive filtering and alternative calculations revolutionizes pulse oximetry sensitivity and specificity during motion and low perfusion," in Proc. 26th Annu. Int. Conf. IEEE Eng. Med. Biol. Soc., San Fransisco, Sep. 2004, vol. 2, pp. 5363-5366.

[7] J. Y. A. Foo, "Comparison of wavelet transformation and adaptive filtering in restoring artifact-induced time-related measurement," Biomed. Signal Process. Control, vol. 1, no. 1, pp. 93-98, 2006.

[8] J. E. Scharf, S. Athan, and D. Cain, "Pulse oximetry through spectral analysis," in Proc. 12th Southern Biomed. Eng. Conf., New Orleans, LA, Apr. 1993, pp. 227-229.

[9] B. S. Kim and S. K. Yoo, "Motion artifact reduction in photoplethysmography using independent component analysis," IEEE Trans. Biomed. Eng., vol. 53, no. 3, pp. 566-568, Mar. 2006.

[10] J. Weng, Z. Ye, and J. Weng, "An improved pre-processing approach for photoplethysmographic signal," in Proc. 27th Annu. Int. Conf. Eng. Med. Biol. Soc., Sep. 2005, pp. 41-44.

[11] M. K. Diab, E. Kiani-Azarbayjany, I. M. Elfadel, R. J. McCarthy, W. M. Weber, and R. A. Smith, "Signal processing apparatus," U.S. Patent 721 598 4, May 2007.

[12] N. Mammone and F.C Morabito, "Independent component analysis and high-order statistics for automatic artifact rejection," in Proc. IEEE Int. Joint Conf. Neural Netw. (IJCNN 2005), Montreal, Quebec, Aug., vol. 4, pp. 2447-2452.

[13] J. Yao and S. Warren, "A short study to assess the potential of independent component analysis for motion artifact separation in wearable pulse oximeter signals," in Proc. 27th Аnnu. Int. Conf. Eng. Med. Biol. Soc., 2005, pp. 3585-3588.

[14] D. Thompson and S. Warren, "A small, high-fidelity reflectance pulse oximeter," presented at the Annual Conf. Exposition, American Society of Engineering Education, Honolulu, Hawaii, Jun. 24-27, 2007.

[15] B. Pilgram, P. Castiglioni, G. Parati, and M. Di Rienzo, "Dynamic detection of rhythmic oscillations in heart-rate tracings: A state-space approach based on fourth-order cumulants," Biol. Cyberern., vol. 76, pp. 299-309, 1997.

[16] J. M. Mendel, "Tutorial on higher-order statistics (spectra) in signal processing and system theory: Theoretical results and some applications," Proc. IEEE, vol. 79, no. 1, pp. 278-305, Jan. 1991.

[17] C. L. Nikias and A. P. Petropulu, Higher-Order Spectra Analysis: A Nonlinear Signal Processing Framework. Englewood Clifs, NJ: PrenticeHall, 1993.

[18] A. Swami, J. M. Mendel, and C. L. Nikias, Higher-Order Spectral Analysis Toolbox User's Guide, R12 for Use With MATLAB ${ }^{\circledR}$. United Signals and Systems, Inc., 2000.

[19] C. L. Nikias and M. R. Raghuveer, "Bispectrum estimation: A digital signal processing framework," Proc. IEEE, vol. 75, no. 7, pp. 867-891, Jul. 1987.

[20] A. Mansour and C. Jutten, "What should we say about the kurtosis?" IEEE Signal Process. Lett., vol. 6, no. 12, pp. 321-322, Dec. 1999.

[21] R. F. Dwyer, "Use of the Kurtosis statistic in the frequency domain as an aid in detecting random signals," IEEE J. Ocean. Eng., vol. 9, no. 2, pp. 85-92, Apr. 1984.

[22] H. Poor, An Introduction to Signal Detection and Estimation, 2nd ed. New York: Springer-Verlag, 1994.

[23] J. Muthuswamy, D. L. Sherman, and N. V. Thakor, "Higher-order spectral analysis of burst patterns in EEG," IEEE Trans. Biomed. Eng., vol. 46, no. 1, pp. 92-99, Jan. 1999.

[24] P. Smaragdis, "Blind separation of convolved mixtures in the frequency domain," presented at the Int. Workshop Independence and Artificial on Neural Networks, University of La Laguna, Tenerife, Spain, Feb. 1998.

[25] A. Hyvrinen, "Fast and robust fixed-point algorithms for independent component analysis," IEEE Trans. Neural Netw., vol. 10, no. 3, pp. 626634, Mar. 1999.

[26] H. M. Park, Y. Jung, T. W. Lee, and S. Y. Lee, "On subband-based blind signal separation for noisy speech recognition," Electron. Lett., vol. 35, pp. 2011-2012, 1999.

[27] E. Bingham and A. Hyvrinen, "A fast fixed-point algorithm for independent component analysis of complex-valued signals," Int. J. Neural Syst., vol. 10 , pp. $1-8,2000$.

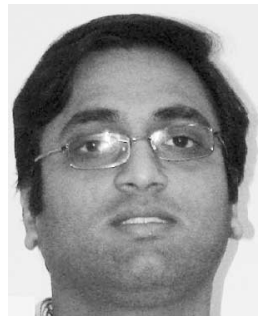

Rajet Krishnan (S'08) received the B.E. degree in electronics and communication engineering from the University of Kerala, India, in 2004, and the M.S degree in electrical and computer engineering from Kansas State University, Manhattan, in 2009.

$\mathrm{He}$ is currently a Research Engineer at the Center of Excellence in Wireless Technology, Indian Institute of Technology Madras, Chennai, India where he is currently engaged in research on investigating interference management techniques in $4 \mathrm{G}$ (LTE-A and $802.16 \mathrm{~m}$ ) wireless systems. From 2004 to 2006, he was a Software Engineer with Infosys Technologies Limited, where he was engaged in research on developing drivers, firmwares for handheld wireless devices. From 2006 to 2009, he was with the Wireless Communication Group, Kansas State University, where he was engaged in research on distributed signal processing and optimization for resource allocation in wireless sensor networks.

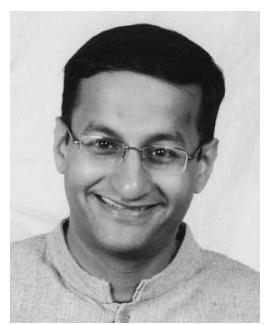

Balasubramaniam (Bala) Natarajan (SM'08) received the B.E degree in electrical engineering from the Birla Institute of Technology and Science, Pilani, India, in 1997, and the Ph.D. degree in electrical engineering from Colorado State University, Fort Collins, in 2002 .

Since Fall 2002, he has been a Faculty Member at the Department of Electrical and Computer Engineering, Kansas State University, Manhattan, where he is currently an Associate Professor and the Director of the Wireless Communication and Information Processing Research Group. In 1997, he was involved in telecommunications research. He is the author or coauthor of numerous papers published in international journals and refereed conference proceedings in the wireless communications and signal processing arena, a book entitled Multi-Carrier Technologies for Wireless Communications (Kluwer, 2002). He is the holder of a patent on customized spreading sequence design algorithm for code-division multiple-access (CDMA) systems. He also has two pending patents on adaptive/customized precoder design for multiple-input and multiple-output systems. His research interests include spread spectrum communications, multi-carrier CDMA and orthogonal frequency-division multiplexing, multiuser detection, cognitive radio networks, sensor signal processing, distributed detection and estimation, and antenna array processing.

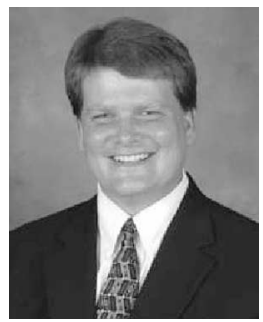

Steve Warren (M'02) received the B.S. and M.S. degrees in electrical engineering from Kansas State University (KSU), Manhattan, in 1989 and 1991, respectively, and the Ph.D. degree in electrical engineering from The University of Texas, Austin, in 1994.

$\mathrm{He}$ is currently an Associate Professor at the Department of Electrical and Computer Engineering, Kansas State University. In August 1999, he was a Principal Member of the Technical Staff, Sandia National Laboratories, Albuquerque, NM. He directs the KSU Medical Component Design Laboratory, a facility supported by the National Science Foundation that provides resources for the research and development of distributed medical monitoring technologies and learning tools that utilize biomedical contexts. His research interests include plug-and-play, point-of-care medical monitoring systems that utilize interoperability standards, wearable sensors and signal processing techniques for the determination of human and animal physiological status, and particle transport simulation applied to radiation hardness analysis of complex assemblies and the determination of photon dose distributions in tissue.

Dr. Warren is a member of the American Society for Engineering Education. 